Supporting Information for

\title{
Naphthalene Benzimidazole Based Neutral Ir(III) Emitters for Deep Red
}

\section{Organic Light-Emitting Diodes}

Palanisamy Rajakannu, ${ }^{\dagger}$ Hyung Suk Kim,${ }^{\dagger}$ Woochan Lee, ${ }^{\dagger}$ Ajay Kumar, ${ }^{\ddagger}$ Min Hyung Lee ${ }^{*, \ddagger}$ and Seunghyup Yoo*,†

†'School of Electrical Engineering, Korea Advanced Institute of Science and Technology (KAIST),

Daejeon 34141, Republic of Korea

Department of Chemistry, University of Ulsan, Ulsan 44610, Republic of Korea

Corresponding Authors

E-mail: $\underline{\text { syoo@ee.kaist.ac.kr (S. Yoo) }}$

E-mail:1mh74@ulsan.ac.kr (M. H. Lee) 


\section{Experimental section}

General discussion. The ligand $\mathrm{L}^{1}$ was synthesized from the 1,2-diaminobenzene using similar procedure reported. ${ }^{1}$ The starting material, 3-bromonaphthalenebenzimidazole was prepared using reported procedure, ${ }^{2}$ while the ligands $\mathrm{L}^{2}$ and $\mathrm{L}^{\mathrm{a}}$ were synthesized from the modified procedure. ${ }^{3}$ The ligands $\mathrm{L}^{\mathrm{a}}$ and $\mathrm{L}^{2}$ were synthesized to confirm the effect of donor unit on the photoluminescence properties to compare with the ligand $\mathrm{L}^{1}$. $\mathrm{L}^{\mathrm{a}}$ possessing 4-tert-butylphenyl unit on the naphthalene unit considered as donor unit. The introduction of donor unit shows slight redshifted emission $\left(512 \mathrm{~nm}\right.$ ) in dichloromethane solution compared to the parent ligand $\mathrm{L}^{1}$ (emission wavelength at $504 \mathrm{~nm}$ in dichloromethane). This red-shift confirms the effect of donor unit on the phenylene unit. Subsequently, we synthesized the ligand $\mathrm{L}^{2}$ possessing diphenylamine (strong donor), which shows strong emission at $608 \mathrm{~nm}$ in solution. The photophysical properties of ligands are shown in Figure S1 and details are given in Table S1. In order to develop the deep-red or near-infrared materials, we have chosen the specific ligands $\mathrm{L}^{1}$ and $\mathrm{L}^{2}$ (without donor and with strong donor respectively) to make the iridium emitters. The ligand $\mathrm{L}^{\mathrm{a}}$ was used only to compare the basic luminescence properties of the ligands.
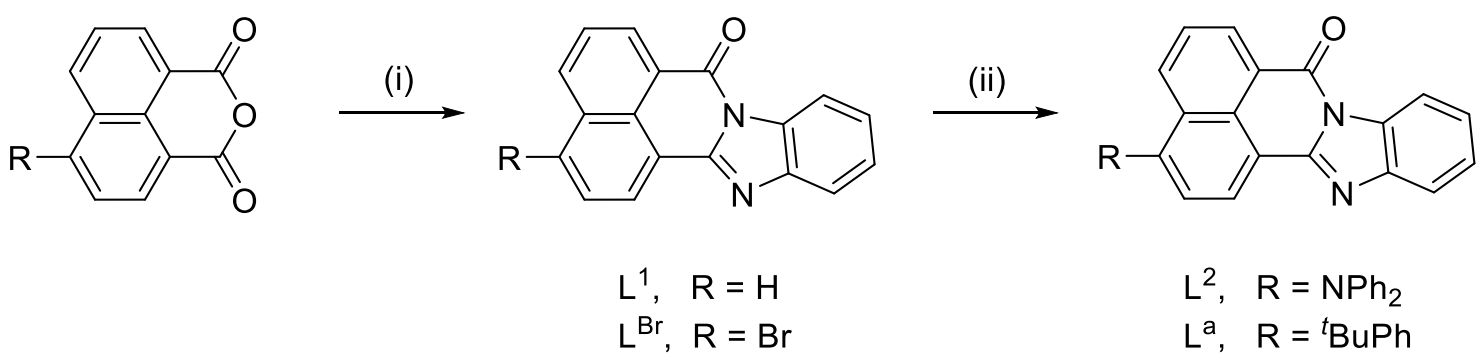

$L^{2}, \quad R=N P h_{2}$

$\mathrm{L}^{\mathrm{a}}, \quad \mathrm{R}={ }^{t} \mathrm{BuPh}$

Scheme S1. Synthesis of NIB based ligands $L^{1}, L^{2}$ and $L^{a}$. conditions: (i) 1,2-diaminobenzene, acetic acid for $\mathrm{L}^{1},{ }^{1} \mathrm{THF}$ for $\mathrm{L}^{\mathrm{Br}}{ }^{2}$ reflux for overnight and (ii) diphenylamine/4-tertbutylphenylboronicacid, $\mathrm{NaO}^{t} \mathrm{Bu}$ and $\mathrm{Pd}\left(\mathrm{PPh}_{3}\right) \mathrm{Cl}_{2}$ in dry toluene, reflux for 12 hours. ${ }^{3}$ 
Synthesis ligand $\mathbf{L}^{\mathrm{a}}$. 4-tert-butylphenylboronic acid (0.61 g, $3.42 \mathrm{mmol}, 1.2$ equi), $\mathrm{NaO}{ }^{t} \mathrm{Bu}(0.55$ $\mathrm{g}, 5.71 \mathrm{mmol}, 2$ equi), 3-bromonaphthalenebenzimidazole $(1 \mathrm{~g}, 2.85 \mathrm{mmol}, 1$ equi) and $\operatorname{Pd}\left(\mathrm{PPh}_{3}\right) \mathrm{Cl}_{2}(0.1 \mathrm{~g}, 014 \mathrm{mmol}, 0.05$ equi) were taken in a Schlenk flask under nitrogen atmosphere and the dry toluene $(50 \mathrm{~mL})$ was added to the mixture. The reaction mixture was allowed to stir at reflux for 24 hours and cooled to room temperature. The resulting mixture was extracted with ethyl acetate $(3 \times 100 \mathrm{~mL})$ and washed with water. The organic layer was separated and dried over anhydrous $\mathrm{Na}_{2} \mathrm{SO}_{4}$. The solvent was removed under reduced pressure and the major product was purified by column chromatography $\left(\mathrm{SiO}_{2}\right.$, ethyl acetate/n-hexane) to give the desired product. Yield: 52\% (0.6 g, $1.48 \mathrm{mmol}) .{ }^{1} \mathrm{H} \mathrm{NMR}\left(\mathrm{CDCl}_{3}, 400 \mathrm{MHz}, \mathrm{ppm}\right): \delta 8.96(\mathrm{~d}, J=7.08 \mathrm{~Hz}, 1 \mathrm{H})$, $8.88(\mathrm{~d}, J=7.56 \mathrm{~Hz}, 1 \mathrm{H}), 8.64-8.61(\mathrm{~m}, 1 \mathrm{H}), 8.49-8.32(\mathrm{~m}, 1 \mathrm{H}), 7.95-7.93(\mathrm{~m}, 1 \mathrm{H}), 7.83-7.77$ (m, 2H), 7.63-7.60 (m, 2H), 7.54-7.51 (m, 4H), $1.46(\mathrm{~s}, 9 \mathrm{H})$.

Synthesis ligand $\mathbf{L}^{2}$. Diphenylamine $\left(0.59 \mathrm{~g}, 3.42 \mathrm{mmol}, 1.2\right.$ equi), $\mathrm{NaO}{ }^{t} \mathrm{Bu}(0.55 \mathrm{~g}, 5.71 \mathrm{mmol}$, 2 equi), 3-bromonaphthalenebenzimidazole (1 g, $2.85 \mathrm{mmol}, 1$ equi) and $\mathrm{Pd}\left(\mathrm{PPh}_{3}\right) \mathrm{Cl}_{2}(0.1 \mathrm{~g}, 014$ mmol, 0.05 equi) were taken in a Schlenk flask under nitrogen atmosphere and the dry toluene (50 $\mathrm{mL}$ ) was added to the mixture. The reaction mixture was allowed to stir at reflux for 12 hours and cooled to room temperature. The resulting mixture was extracted with ethyl acetate $(3 \times 100 \mathrm{~mL})$ and washed with water. The organic layer was separated and dried over anhydrous $\mathrm{Na}_{2} \mathrm{SO}_{4}$. The solvent was removed under reduced pressure and the major product was purified by column chromatography $\left(\mathrm{SiO}_{2}\right.$, ethyl acetate/n-hexane) to give the desired product. Yield: $38 \%(0.47 \mathrm{~g}$, $1.08 \mathrm{mmol}$ ). ${ }^{1} \mathrm{H}$ NMR (DMSO- $\left.d_{6}, 400 \mathrm{MHz}, \mathrm{ppm}\right): \delta 8.91(\mathrm{br}, 1 \mathrm{H}), 8.69(\mathrm{~d}, J=8.12 \mathrm{~Hz}, 1 \mathrm{H})$, 8.58-8.56 (m, 1H), $8.17(\mathrm{~d}, J=8.12 \mathrm{~Hz}, 1 \mathrm{H}), 7.92-7.90(\mathrm{~m}, 1 \mathrm{H}), 7.56(\mathrm{t}, 1 \mathrm{H}), 7.52-7.47(\mathrm{~m}, 2 \mathrm{H})$, $7.42(\mathrm{~d}, J=8.12 \mathrm{~Hz}, 1 \mathrm{H}), 7.29-7.24(\mathrm{~m}, 4 \mathrm{H}), 7.1-7.05(\mathrm{~m}, 6 \mathrm{H})$. 
Table S1. Photophysical data of ligands $\mathrm{L}^{1}, \mathrm{~L}^{2}$ and $\mathrm{L}^{\mathrm{a}}$

\begin{tabular}{clccc}
\hline Ligands & \multicolumn{1}{c}{$\boldsymbol{\lambda}_{\text {abs }}(\mathbf{n m})^{a}$} & $\lambda_{\mathbf{P L}}(\mathbf{n m})^{b}$ & $\boldsymbol{\Phi}_{\mathbf{P L}}{ }^{b}$ & $\langle\tau\rangle(\mathbf{n s})^{c}$ \\
\hline $\mathrm{L}^{1}$ & $228,255,295,331,387$ & 504 & 0.98 & 9.55 \\
$\mathrm{~L}^{\mathrm{a}}$ & $226,259,284,389,483,539$ & 512 & 1.03 & 7.97 \\
$\mathrm{~L}^{2}$ & $227,262,301,422$ & 608 & 0.21 & 6.45
\end{tabular}

${ }^{a}$ Measured in $10^{-5} \mathrm{M}$ solution in $\mathrm{CH}_{2} \mathrm{Cl}_{2}$ at room temperature. ${ }^{b}$ Absolute PLQYs, Measured in $10^{-}$ ${ }^{5} \mathrm{M}$ solution in degassed $\mathrm{CH}_{2} \mathrm{Cl}_{2}$ at room temperature. ${ }^{c}$ The fitted PL lifetimes from degassed $\mathrm{CH}_{2} \mathrm{Cl}_{2}$ at room temperature.

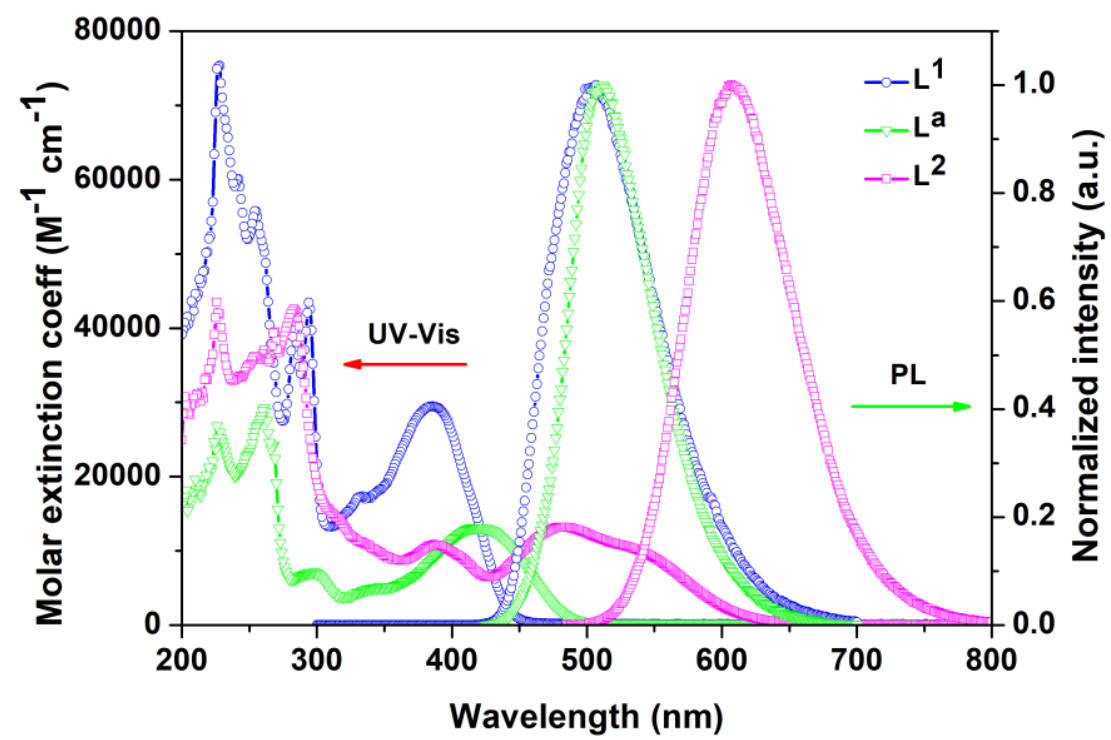

Figure S1. Absorption and emission spectra of $\mathrm{L}^{1}\left(\lambda_{\mathrm{ex}}=385 \mathrm{~nm}\right), \mathrm{L}^{\mathrm{a}}\left(\lambda_{\mathrm{ex}}=420 \mathrm{~nm}\right)$ and $\mathrm{L}^{2}\left(\lambda_{\mathrm{ex}}=\right.$ $480 \mathrm{~nm}$ ) in degassed dichloromethane solution at RT. 


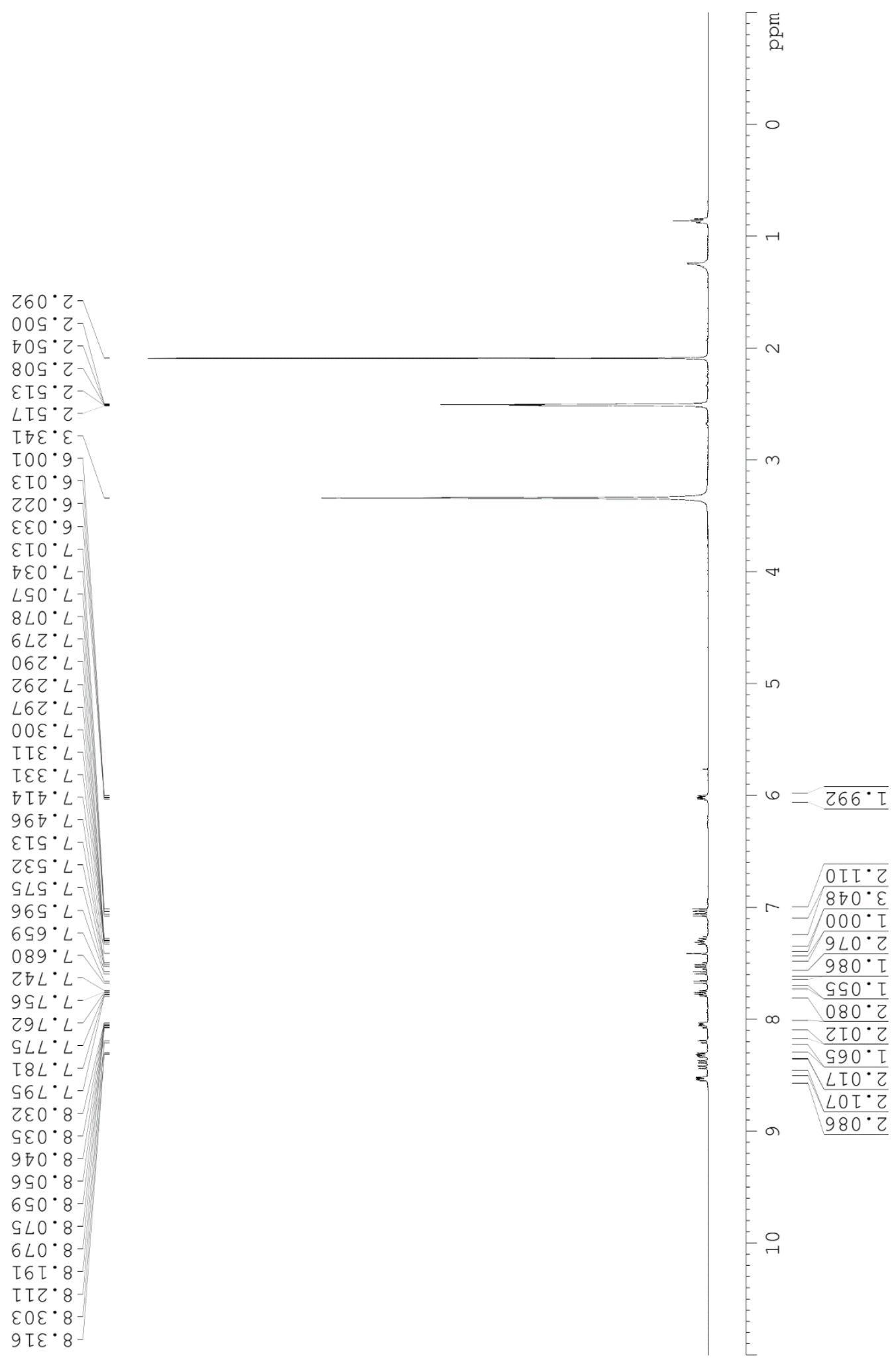

Figure S2. ${ }^{1} \mathrm{H}$ NMR spectrum of 1 in DMSO- $d_{6}$ at room temperature. 


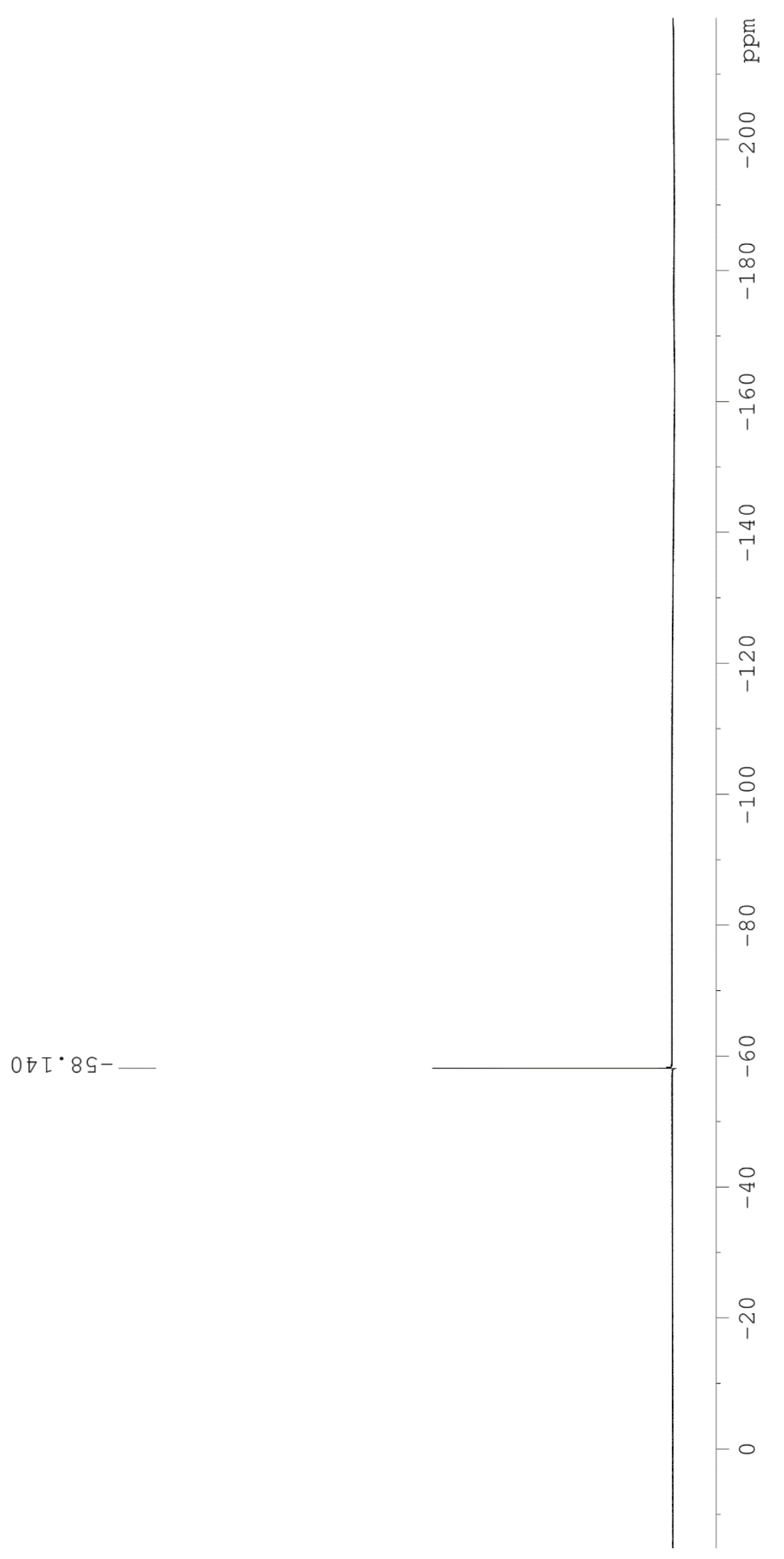

Figure S3. ${ }^{19} \mathrm{~F}$ NMR spectrum of $\mathbf{1}$ in DMSO- $d_{6}$ at room temperature. 


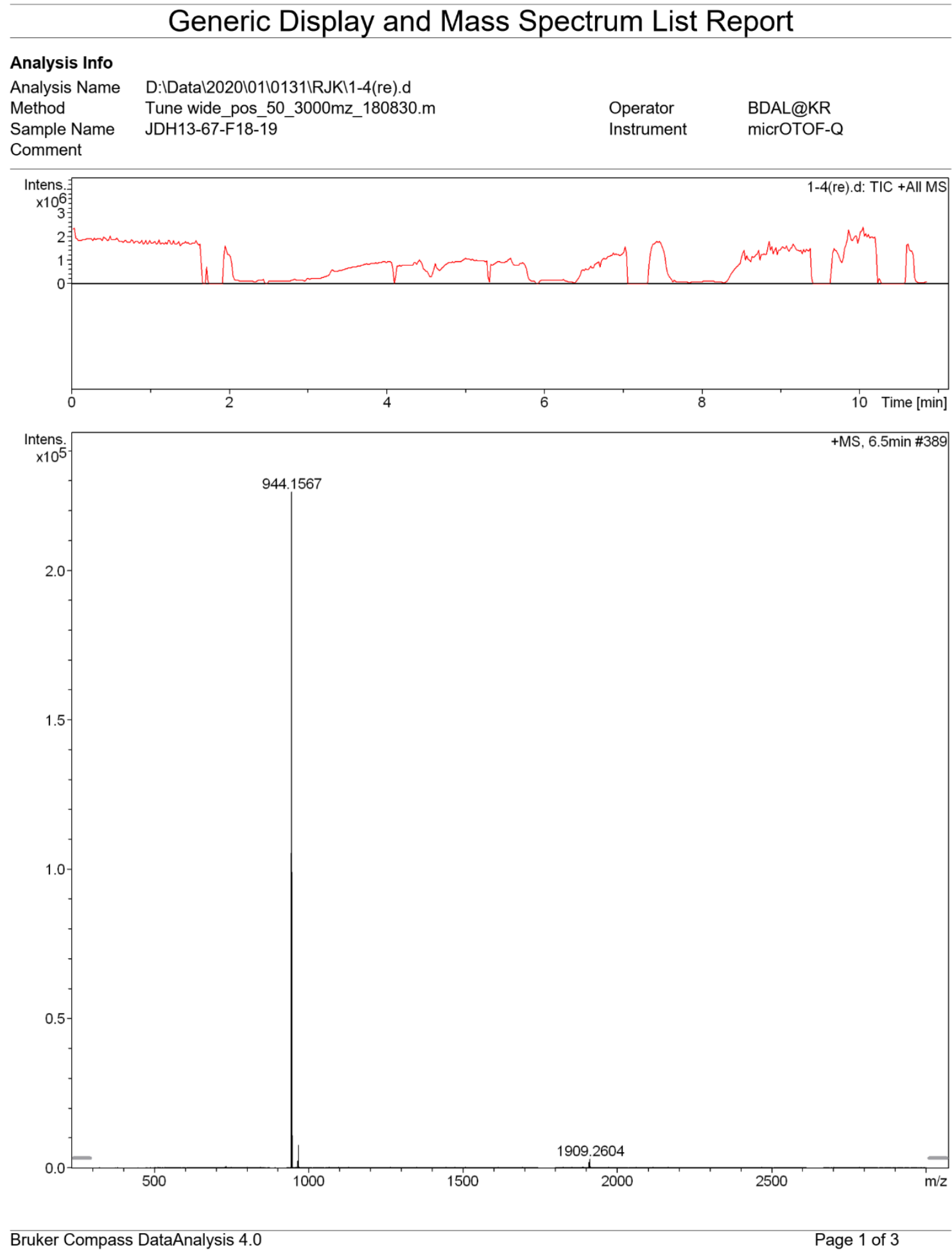

Figure S4. MS spectrum of 1 in dichloromethane at room temperature. 


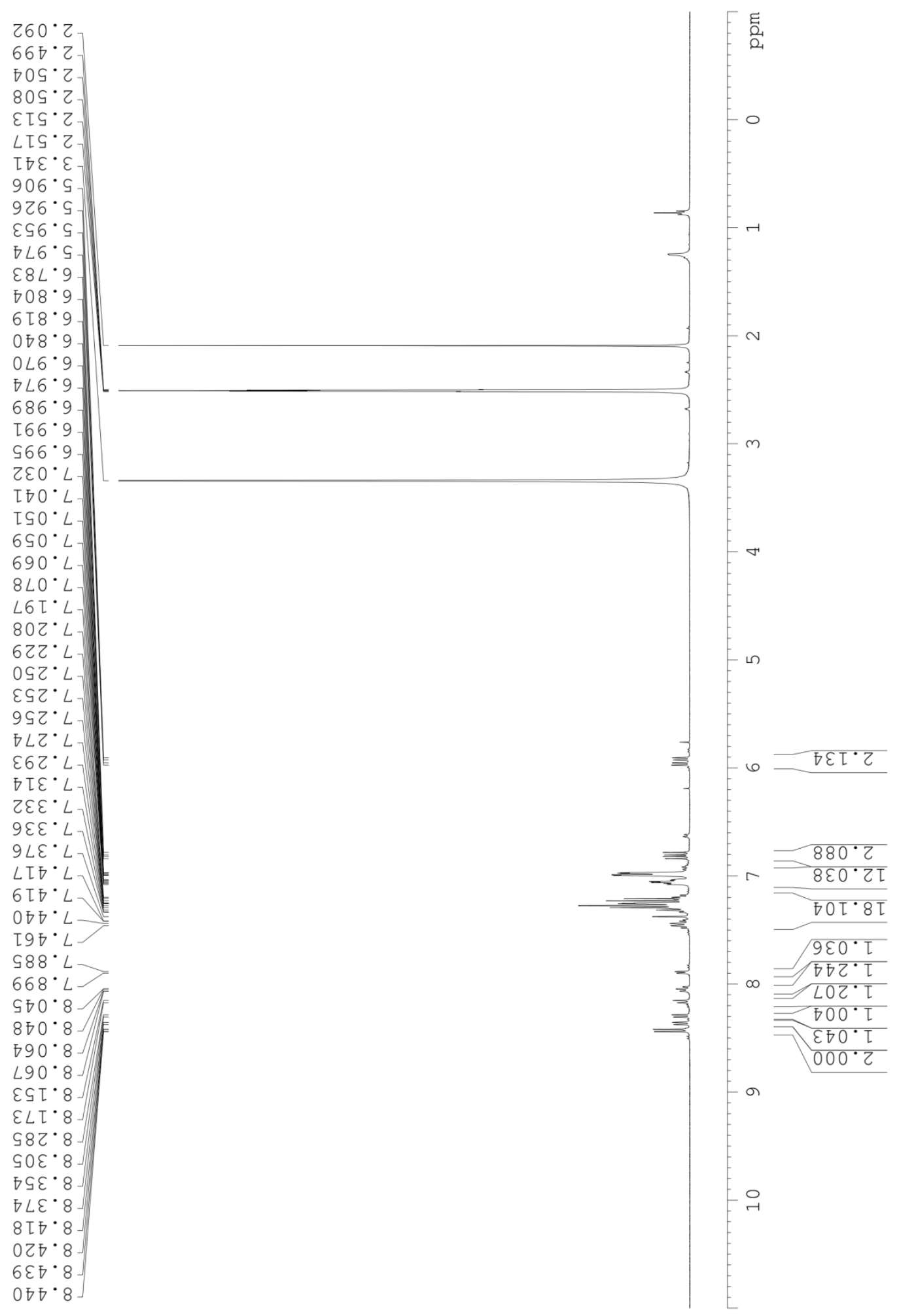

Figure S5. ${ }^{1} \mathrm{H}$ NMR spectrum of 2 in DMSO- $d_{6}$ at room temperature. 


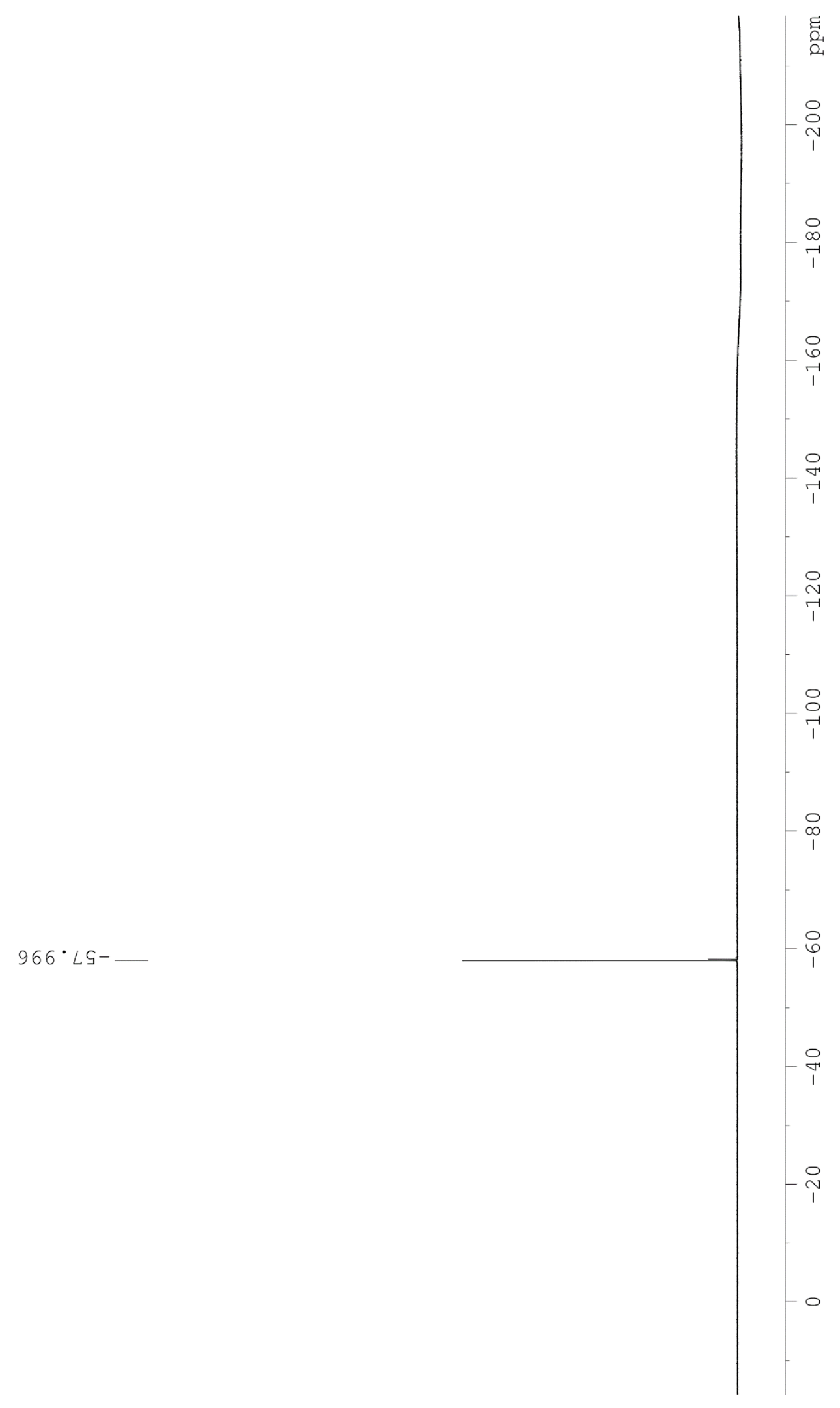

Figure S6. ${ }^{19} \mathrm{~F}$ NMR spectrum of 2 in DMSO- $d_{6}$ at room temperature. 


\section{Generic Display and Mass Spectrum List Report}

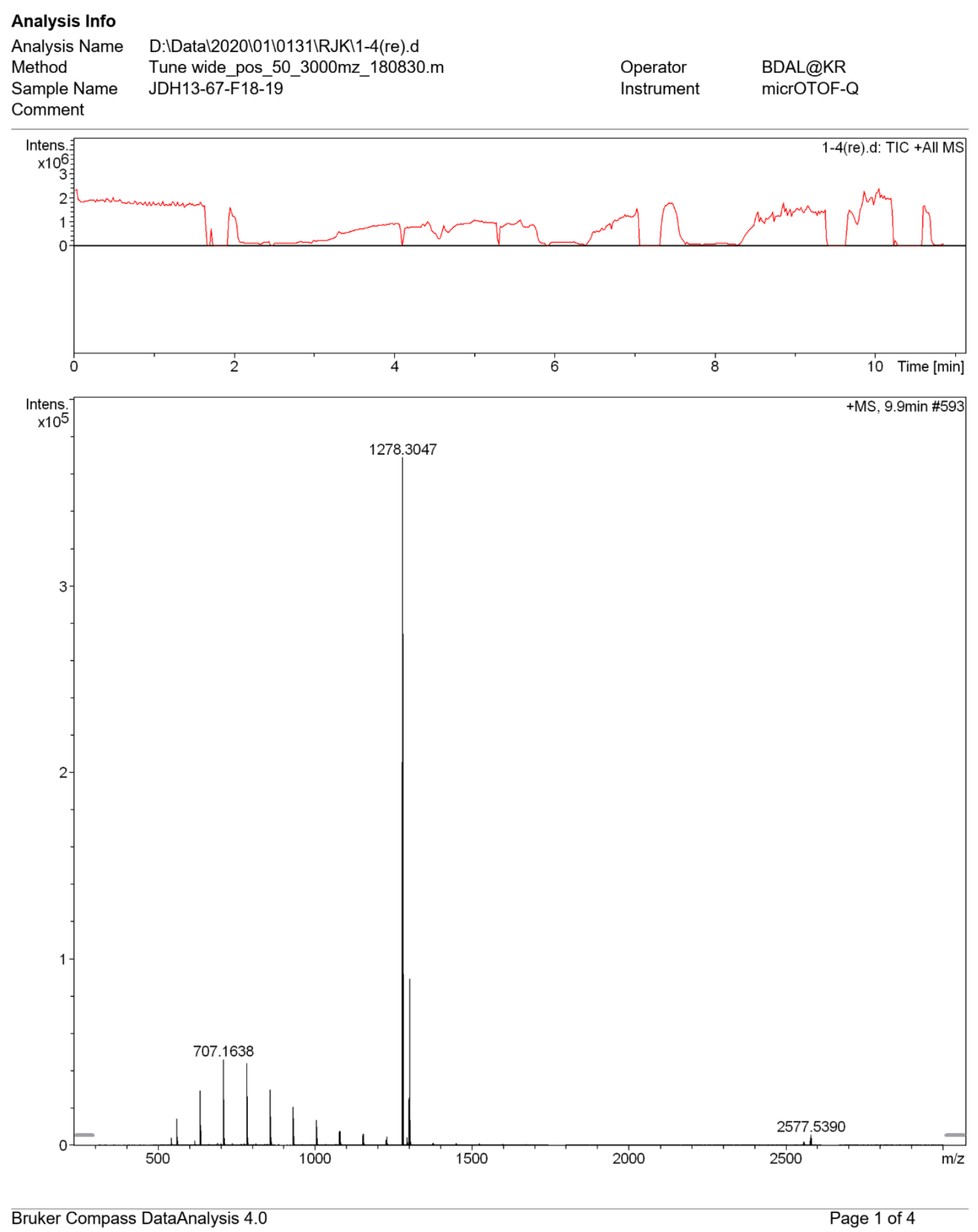

Figure S7. MS spectrum of $\mathbf{2}$ in dichloromethane at room temperature. 


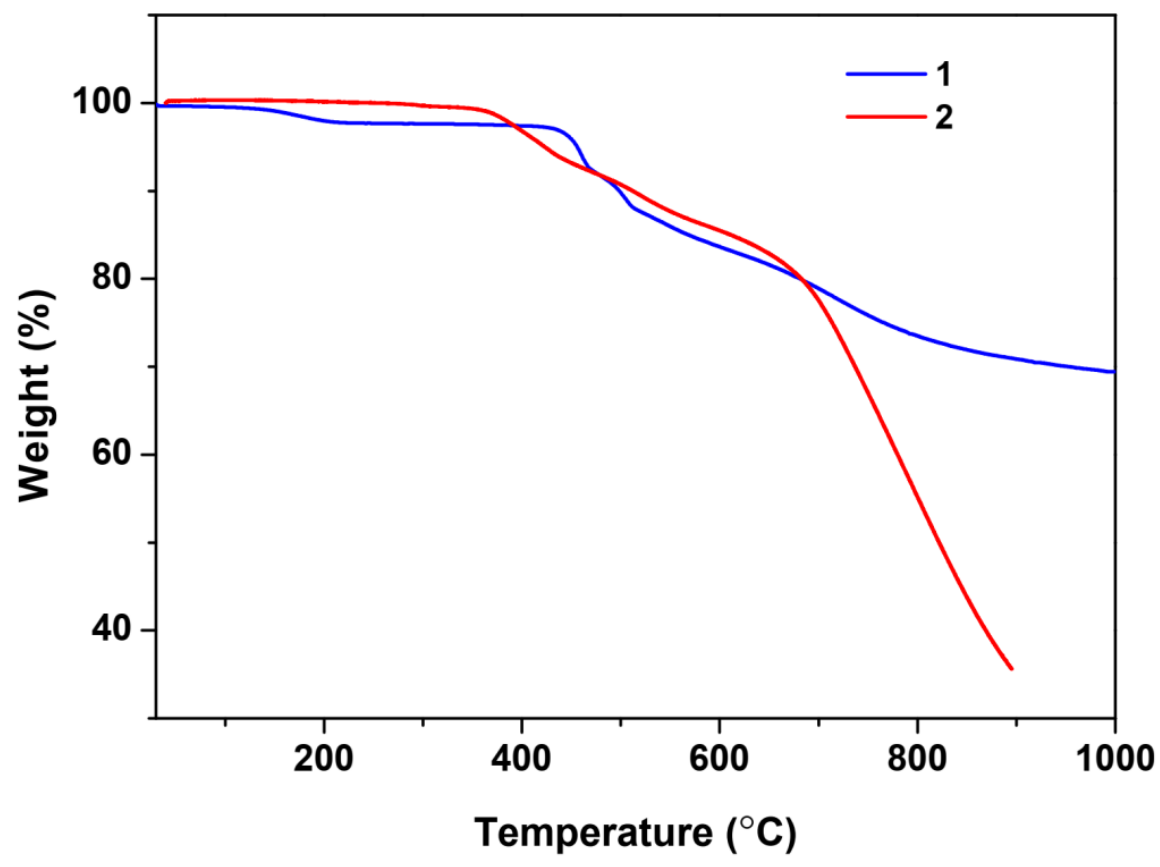

Figure S8. TGA curves of $\mathbf{1}$ and $\mathbf{2}$ under $\mathrm{N}_{2}$ atmosphere.

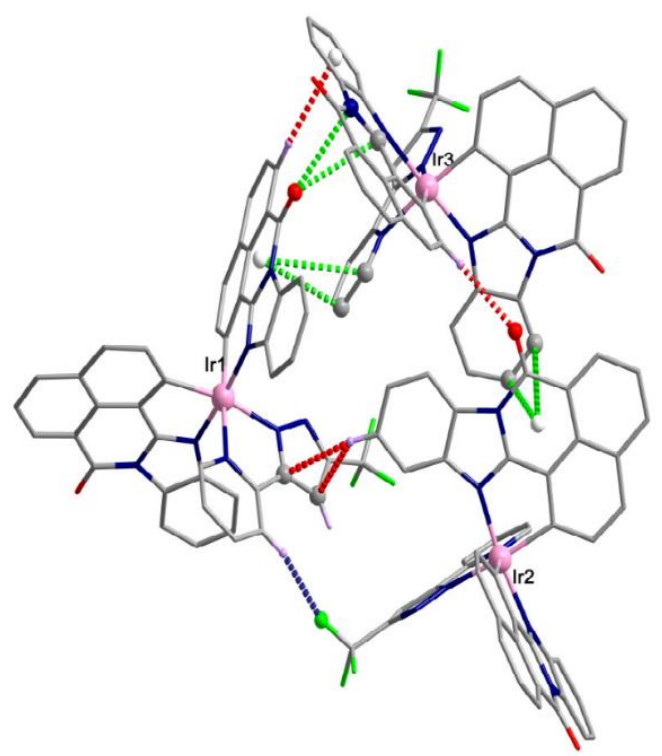

Figure S9. Intermolecular C-H $\cdots \pi$ and $\pi \cdots \pi$ interactions in $\mathbf{1}$. 
Table S2. Crystallographic data and parameters of $\mathbf{1 .}$

\begin{tabular}{|c|c|}
\hline Compound & 1 \\
\hline Indentification code & SY-RK-Ir5 \\
\hline Formula & $\mathrm{C}_{45} \mathrm{H}_{23} \mathrm{~F}_{3} \mathrm{IrN}_{7} \mathrm{O}_{2}$ \\
\hline Formula weight & 942.90 \\
\hline Temperature (K) & $223(2)$ \\
\hline Wavelength, (̊) & 0.71073 \\
\hline Crystal system & Triclinic \\
\hline Space group & P-1 \\
\hline$a(\AA)$ & $16.5008(13)$ \\
\hline$b(\AA)$ & $19.9670(14)$ \\
\hline$c(\AA)$ & 21.3147(19) \\
\hline$\alpha\left(^{\circ}\right)$ & $101.966(4)$ \\
\hline$\beta\left(^{\circ}\right)$ & $102.397(4)$ \\
\hline$\gamma\left({ }^{\circ}\right)$ & $106.667(3)$ \\
\hline Volume $\left(\AA^{3}\right)$ & $6293.5(9)$ \\
\hline$Z$ & 6 \\
\hline $\mathrm{D}($ calcd $), \mathrm{Mg} / \mathrm{m}^{3}$ & 1.493 \\
\hline Cryst. size, $\mathrm{mm}^{3}$ & $0.27 \times 0.16 \times 0.14$ \\
\hline abs coeff, $\mathrm{mm}^{-1}$ & 3.240 \\
\hline Data/restraints/parameters & $22151 / 534 / 1678$ \\
\hline Goodness-of-fit on $\mathrm{F}^{2}$ & 1.083 \\
\hline $\mathrm{R}_{1}[I>2 \sigma(I)]$ & 0.0269 \\
\hline $\mathrm{R}_{2}[I>2 \sigma(I)]$ & 0.0890 \\
\hline
\end{tabular}


Table S3. Selected structural parameters observed in $\mathbf{1 .}$

\begin{tabular}{|c|c|c|c|}
\hline \multicolumn{4}{|c|}{ Bond lengths $(\AA)$} \\
\hline Ir1-N1 & $2.131(4)$ & Ir2-N13 & $2.082(4)$ \\
\hline Ir1-N2 & $2.090(4)$ & Ir2-C63 & $2.024(5)$ \\
\hline Ir1-N4 & $2.088(4)$ & Ir2-C81 & $2.044(4)$ \\
\hline Ir1-N6 & $2.071(4)$ & Ir3-N15 & $2.136(4)$ \\
\hline Ir1-C18 & $2.053(5)$ & Ir3-N16 & $2.085(4)$ \\
\hline Ir1-C36 & $2.024(4)$ & Ir3-N18 & $2.079(4)$ \\
\hline Ir2-N8 & $2.147(4)$ & Ir3-N20 & $2.084(4)$ \\
\hline Ir2-N9 & $2.076(4)$ & Ir3-C108 & $2.032(4)$ \\
\hline Ir2-N11 & $2.088(4)$ & Ir3-C126 & $2.046(5)$ \\
\hline \multicolumn{4}{|c|}{ Bond angles $\left({ }^{\circ}\right)$} \\
\hline N1-Ir1-C36 & $174.4(1)$ & N11-Ir2-N13 & $173.6(2)$ \\
\hline N2-Ir1-C18 & $168.3(2)$ & N15-Ir3-C108 & $172.9(2)$ \\
\hline N4-Ir1-N6 & $174.3(1)$ & N16-Ir3-C126 & $171.1(2)$ \\
\hline N8-Ir2-C63 & $170.0(2)$ & N18-Ir3-N20 & $169.4(2)$ \\
\hline N9-Ir2-C81 & $171.6(2)$ & & \\
\hline
\end{tabular}


Table S4. Comparison of bond lengths and angles of $\mathbf{1 .}$

\section{Bond lengths $(\AA)$}

\begin{tabular}{cccc}
\hline \multicolumn{2}{r}{ Single-crystal Structure } & \multicolumn{2}{c}{ Optimized Structure } \\
\hline Ir1-N1 & $2.131(4)$ & Ir1-N1 & 2.214 \\
Ir1-N2 & $2.090(4)$ & Ir1-N2 & 2.121 \\
Ir1-N4 & $2.088(4)$ & Ir1-N4 & 2.122 \\
Ir1-N6 & $2.071(4)$ & Ir1-N6 & 2.108 \\
Ir1-C18 & $2.053(5)$ & Ir1-C18 & 2.063 \\
Ir1-C36 & $2.024(4)$ & Ir1-C36 & 2.040 \\
\hline
\end{tabular}
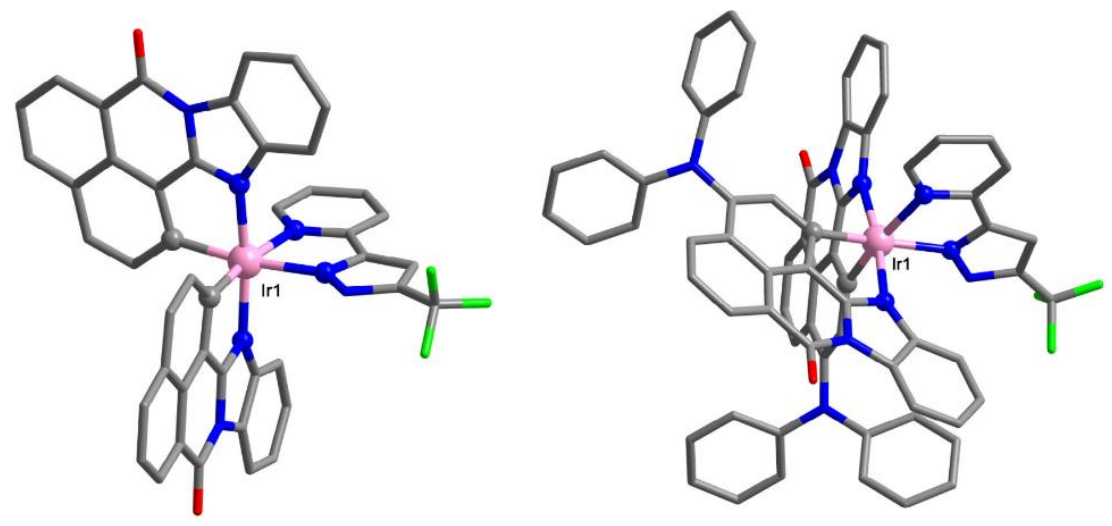

Figure S10. Optimized geometries of 1 and $\mathbf{2}$ in the ground states at B3LYP/6-31G(d) and LANL2DZ with ECP for Ir atom (DFT level). 
Table S5. Experimental and calculated energy values of $\mathbf{1}$ and $\mathbf{2}$.

\begin{tabular}{|c|c|c|c|c|c|}
\hline & $\begin{array}{c}\lambda_{\mathrm{em}} \\
{\left[\mathrm{T}_{1} \rightarrow \mathrm{S}_{0}\right]} \\
(\text { Exp. }) \\
{[\text { solution] }} \\
(\mathrm{nm}, \mathrm{eV})\end{array}$ & $\begin{array}{c}\lambda_{\text {em }} \\
{\left[\mathrm{T}_{1}\left(\mathrm{~T}_{2}\right) \rightarrow \mathrm{S}_{0}\right]} \\
(\mathrm{Sim} .) \\
{[\mathrm{gas}]} \\
(\mathrm{nm}, \mathrm{eV})\end{array}$ & $\begin{array}{c}\lambda_{\mathrm{em}} \\
{\left[\mathrm{T}_{1}\left(\mathrm{~T}_{2}\right) \rightarrow \mathrm{S}_{0}\right]} \\
(\mathrm{Sim} .) \\
{[\text { solution }]^{\mathrm{a}}} \\
(\mathrm{nm}, \mathrm{eV})\end{array}$ & $\begin{array}{c}\lambda_{\text {abs }} \\
{\left[\mathrm{S}_{0} \rightarrow \mathrm{S}_{1}\right]} \\
(\text { Exp. }) \\
{[\text { solution] }} \\
(\mathrm{nm}, \mathrm{eV})\end{array}$ & $\begin{array}{c}\lambda_{\text {abs }} \\
{\left[\mathrm{S}_{0} \rightarrow \mathrm{S}_{1}\right]} \\
(\mathrm{Sim} .) \\
{[\mathrm{gas}]} \\
(\mathrm{nm}, \mathrm{eV})\end{array}$ \\
\hline 1 & $635,1.95$ & $\begin{array}{l}702(675), \\
1.77(1.84)\end{array}$ & $\begin{array}{l}689(673), \\
1.80(1.84)\end{array}$ & $506,2.45$ & $505,2.46$ \\
\hline 2 & $655,1.89$ & $\begin{array}{l}903(724), \\
1.37(1.71)\end{array}$ & $\begin{array}{l}896(722), \\
1.38(1.72)\end{array}$ & $518,2.39$ & $595,2.08$ \\
\hline
\end{tabular}

${ }^{a}$ The solvent effect (DCM) was considered on the basis of polarizable continuum model (PCM) using the integral equation formalism variant (IEFPCM $)^{4,5}$ for the calculations. 


\section{NTOs @ $\mathrm{T}_{2}$ (1)}

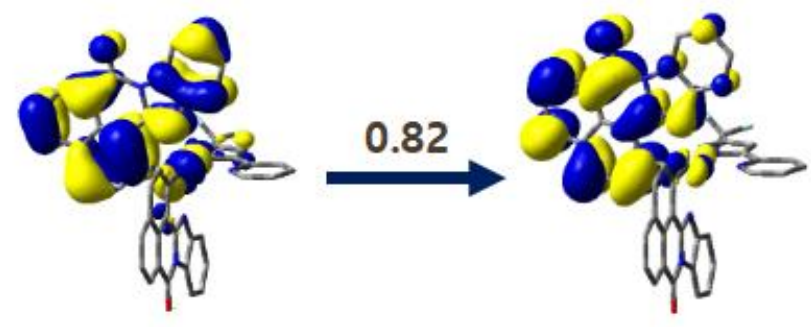

HONTO

\section{LUNTO}

\section{NTOs @ $\mathrm{T}_{2}(2)$}

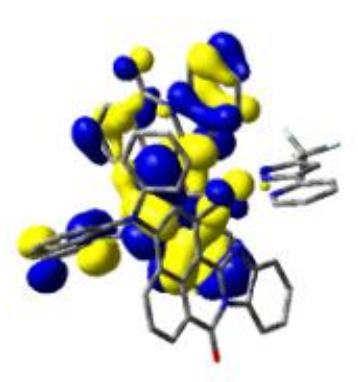

HONTO
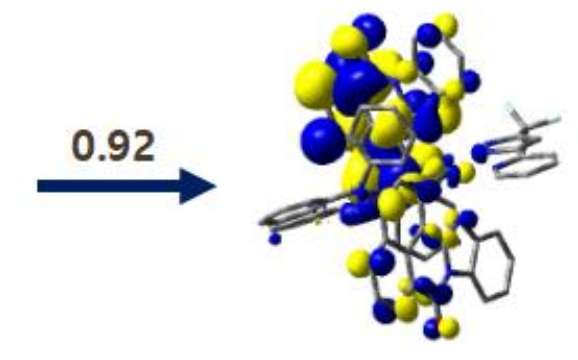

LUNTO

Figure S11. Natural transition orbitals (NTOs) pairs for $T_{2}$ state at $T_{1}$ optimized geometry using the DFT level [UB3LYP/6-31G(d) and LANL2DZ with ECP for Ir]. 


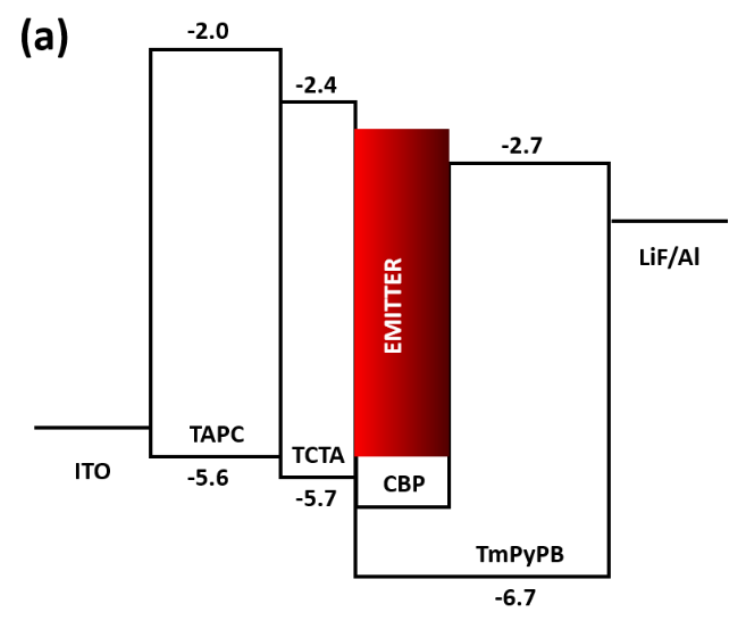

(b)

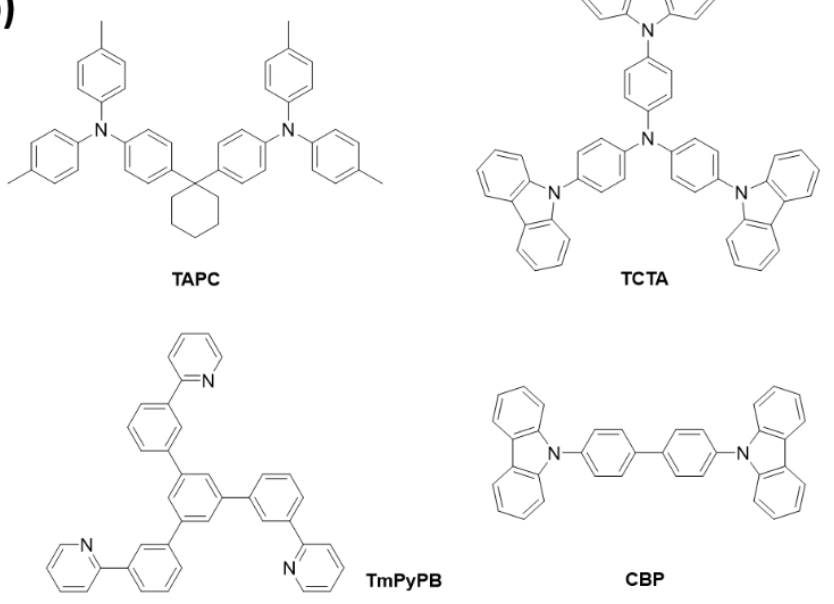

Figure S12. (a) Energy level diagrams of each layer in the device (in eV) relative to the vacuum level. and (b) structural drawings of the utilized materials in this study. 
Table S6. Device performance of the fabricated PHOLED with the emitter 2.

\begin{tabular}{|c|c|c|c|c|c|c|}
\hline \multicolumn{7}{|c|}{ Device Performance (Emitter 2) } \\
\hline Emitter & $\begin{array}{c}\lambda_{\mathrm{EL}} \\
{[\mathrm{nm}]}\end{array}$ & $\operatorname{CIE}(x, y)^{a}$ & $\begin{array}{l}V_{\text {on }}^{\text {b }} \\
{[\mathbf{V}]}\end{array}$ & $\begin{array}{c}\text { Max. luminance } \\
{\left[\mathbf{c d} / \mathbf{m}^{2}\right]}\end{array}$ & $\begin{array}{c}\text { Max. } \\
\text { radiance } \\
{\left[\mathrm{W} / \mathbf{m}^{2} / \mathbf{s r}\right]}\end{array}$ & $\begin{array}{c}\eta_{\mathrm{EQE}}^{\mathrm{c}, \mathrm{d}, \mathrm{e}} \\
{[\%]}\end{array}$ \\
\hline $\begin{array}{c}2 \\
(5 \text { wt. } \%)\end{array}$ & 656,721 & $\begin{array}{l}0.579, \\
0.406)\end{array}$ & 3.8 & 800 & 10.7 & $7.9^{\mathrm{c}}, 4.7^{\mathrm{d}}, \mathrm{N} / \mathrm{A}$ \\
\hline $\begin{array}{c}2 \\
(10 \text { wt. \%) }\end{array}$ & 657,722 & $\begin{array}{l}(0.609 \\
0.315)\end{array}$ & 4.4 & 1500 & 38.2 & $6.9^{\mathrm{c}}, 3.9^{\mathrm{d}}, 2.0^{\mathrm{e}}$ \\
\hline
\end{tabular}

${ }^{a}$ Color coordinates (CIE 1931) at maximum luminance; ${ }^{b} V_{\text {on }}$ : Applied voltage at the luminance of $1 \mathrm{~cd} / \mathrm{m}^{2} ;$ External quantum efficiency at ${ }^{\mathrm{c}}$ maxium, ${ }^{\mathrm{d}} 100 \mathrm{~cd} / \mathrm{m}^{2}$, and ${ }^{\mathrm{e}} 1,000 \mathrm{~cd} / \mathrm{m}^{2}$.

Table S7. Device performance of fabricated PHOLED at various doping conditions.

\begin{tabular}{|c|c|c|c|c|c|c|}
\hline \multicolumn{7}{|c|}{ Device Performance } \\
\hline Emitter & $\eta_{\mathrm{PE}^{\mathrm{a}}}$ & $\eta_{\mathrm{PE}}{ }^{\mathrm{b}}$ & $\eta_{\mathrm{PE}}{ }^{\mathrm{c}}$ & $\eta_{\mathrm{CE}}^{\mathrm{d}}$ & $\eta_{\mathrm{CE}}{ }^{\mathrm{e}}$ & $\eta_{\mathrm{CE}}^{\mathrm{f}}$ \\
\hline & {$[\mathrm{Im} / \mathbf{W}]$} & {$[\mathrm{lm} / \mathrm{W}]$} & {$[\mathrm{lm} / \mathbf{W}]$} & {$[\%]$} & [\%] & [\%] \\
\hline $\begin{array}{c}\mathbf{1} \\
(5 \text { wt. \%) }\end{array}$ & 3.2 & 2.2 & 0.8 & 3.0 & 2.6 & 1.3 \\
\hline $\begin{array}{c}\mathbf{2} \\
(5 \text { wt. \%) }\end{array}$ & 3.9 & 1.8 & N/A & 3.7 & 2.2 & N/A \\
\hline $\begin{array}{c}\mathbf{2} \\
(10 \text { wt. \%) }\end{array}$ & 1.4 & 0.5 & 0.2 & 1.6 & 0.9 & 0.5 \\
\hline
\end{tabular}

Power efficiency at a maxium, ${ }^{\mathrm{b}} 100 \mathrm{~cd} / \mathrm{m}^{2}$, and ${ }^{\mathrm{c}} 1,000 \mathrm{~cd} / \mathrm{m}^{2}$; Current efficiency at ${ }^{\mathrm{d}}$ maxium, ${ }^{\mathrm{e}} 100$ $\mathrm{cd} / \mathrm{m}^{2}$, and ${ }^{\mathrm{f}} 1,000 \mathrm{~cd} / \mathrm{m}^{2}$. 
(a)

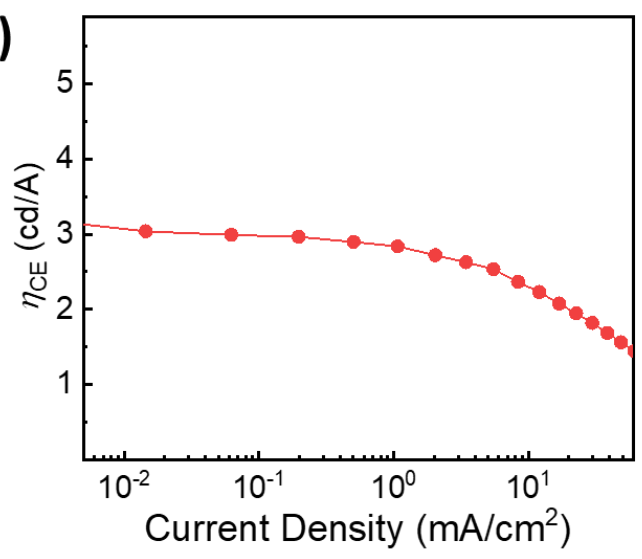

(b)

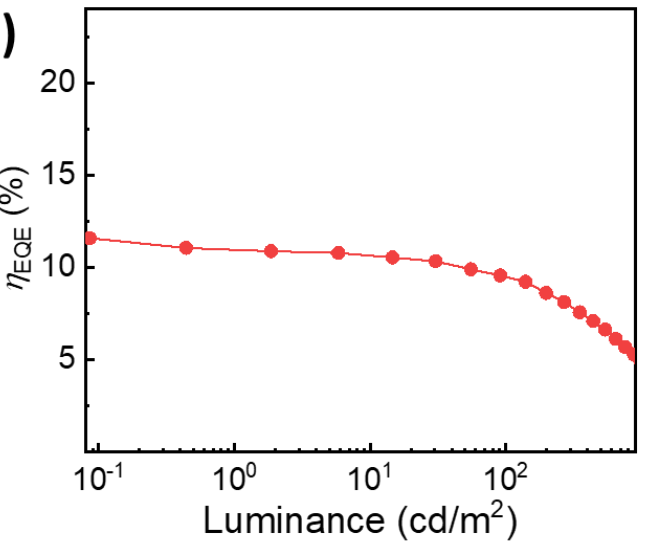

Figure S13. (a) Current efficiency-current density $\left(\eta_{\mathrm{CE}}-J\right)$ characteristics of device with emitter 1 (@ 5 wt. \%). (b) External quantum efficiency-luminance $\left(\eta \eta_{\mathrm{EQE}}-L\right)$ characteristics of the tested device. 

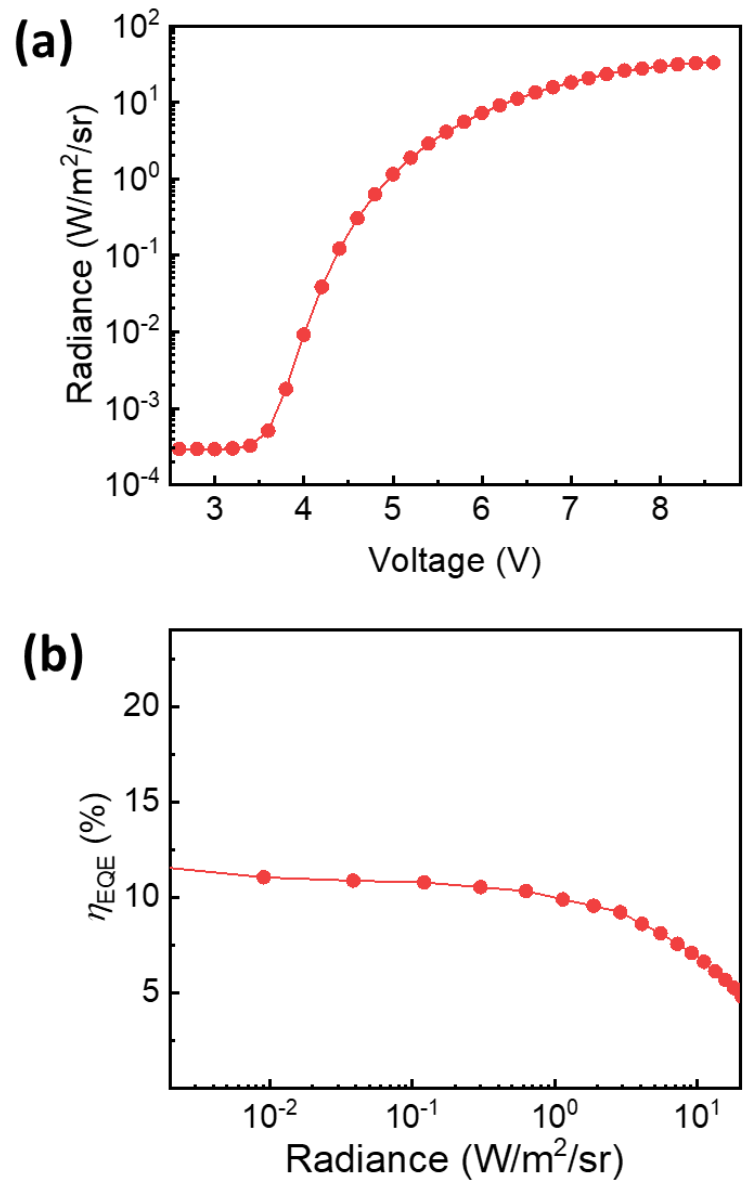

Figure S14. (a) Radiance-voltage $(R-V)$ characteristics of device with emitter 1. (b) External quantum efficiency-radiance $\left(\eta_{\mathrm{EQE}}-R\right)$ characteristics of the tested device. 
(a)

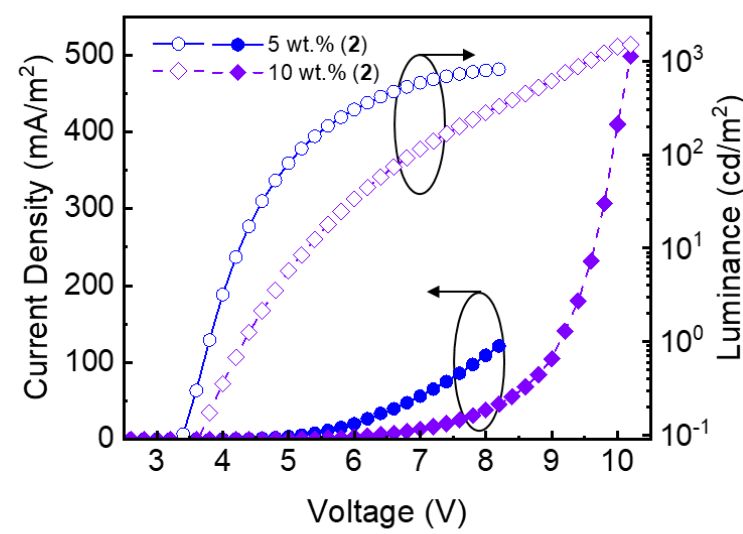

(b)

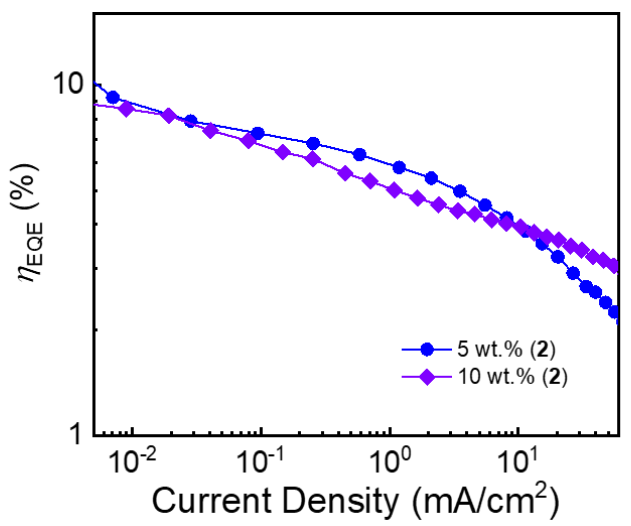

(c)

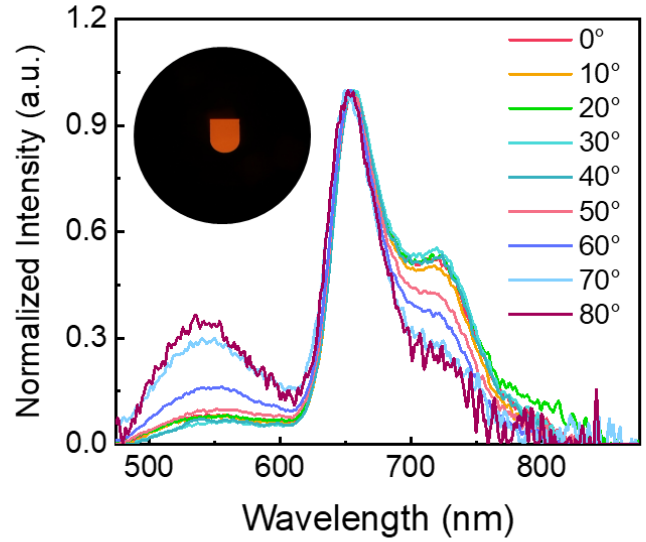

(d)

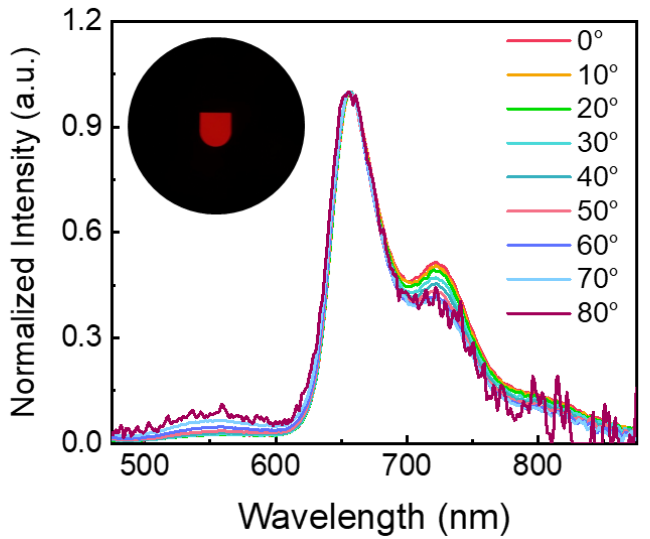

Figure S15. (a) Current density-voltage-luminance $(J-V-L)$ characteristics of device with emitter

2. (b) External quantum efficiency-current density $\left(\eta_{\mathrm{EQE}}-J\right)$ characteristics of device with emitter

2. (c and d) Normalized angular spectrum of the fabricated PHOLED and photograph of working device with emitter 2 (@ 5 wt.\% and 10 wt.\%). 


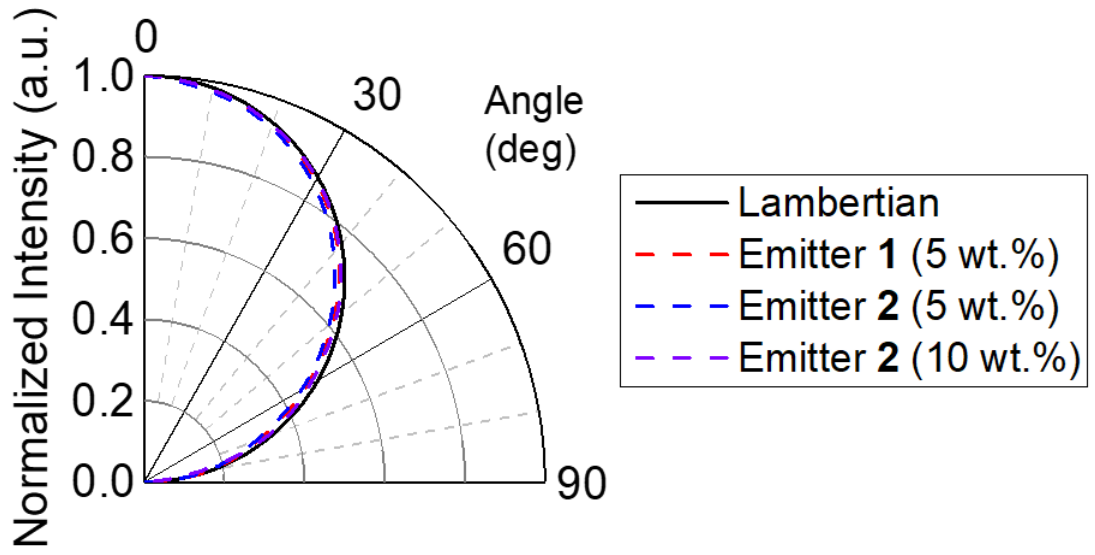

Figure S16. Normalized angular electroluminescence (EL) intensity for a series of devices. 


\section{Comparison of electroluminescence properties}

Recently, the organic bidentate ligand with extended aromatic rings and strong donor units (thiophene and its derivatives) were utilized to make deep red and NIR based the iridium emitters. ${ }^{6-}$

${ }^{11}$ In the present study, we have demonstrated the utilization of rigid emitter $\mathbf{1}$ for the fabrication of deep red OLED. The emitter 1 has no heavy/bulky substituents (donor or acceptor) on the framework which gives a short radiative lifetime with emission wavelength $637 \mathrm{~nm}$ (PL) and moderate PLQY. The PHOLED fabrication of 1 gives better performance (10.9\%) with EL $\lambda_{\max }$ 644 and $700 \mathrm{~nm}$. Compared to the reported pyridine/imidazole and its derivative based iridium complexes (rigid/non-rigid framework) which shows moderate external quantum efficiency in the deep red and NIR region, the emitter 1 show relatively better and comparable PHOLED performance and shown in Scheme S2. ${ }^{6-11}$ This study shows the importance of rigid bidentate organic ligand in making deep red or NIR based iridium emitters with a suitable ancillary ligand.
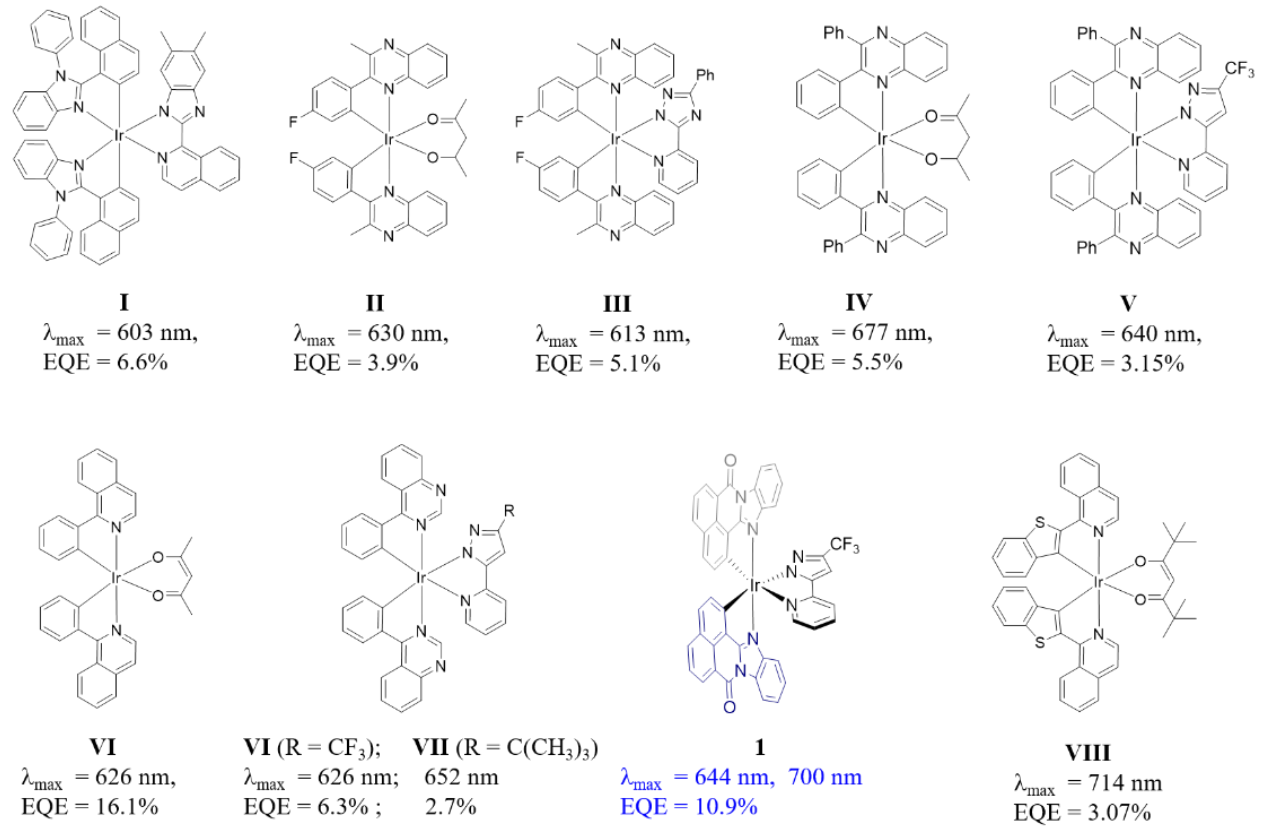

Scheme S2. Structure and EL properties of iridium complexes I-VIII and $\mathbf{1 .}$ 


\section{References}

(1) Zhang, K.; Dai, Y.; Zhang, X.; Xiao, Y. Synthesis and Photophysical Properties of Three Ladder-Type Chromophores with Large and Rigid Conjugation Structures. Dyes and Pigments 2014, 102, 1-5.

(2) Verma, M.; Luxami, V.; Paul, K. Synthesis, in Vitro Evaluation and Molecular Modelling of Naphthalimide Analogue as Anticancer Agents. Eur. J. Med. Chem. 2013, 68, 352-360.

(3) Jiang, W.; Tang, J.; Qi, Q.; Sun, Y.; Ye, H.; Fu, D. An Experimental and Computational Study of Intramolecular Charge Transfer: Diarylamino Derivatives of $7 \mathrm{H}$ Benzimidazo(2,1-a)Benz(d,e)isoquinolin-7-ones. 2009, 80, 279-286.

(4) Cances, E.; Mennucci, B.; Tomasi, J. A New Integral Equation Formalism for the Polarizable Continuum Model: Theoretical Background and Applications to Isotropic and Anisotropic Dielectrics. J. Chem. Phys. 1997, 107, 3032-3041.

(5) Mennucci, B.; Tomasi, J. Evaluation of Solvent Effects in Isotropic and Anisotropic Dielectrics and in Ionic Solutions with a Unified Integral Equation Method: Theoretical Bases, Computational Implementation, and Numerical Applications. J. Phys. Chem. B 1997, 101, 10506-10517.

(6) Cao, H. -T.; Ding, L.; Yu, J.; Shan, G. -G.; Wang, T.; Sun, H. -Z.; Gao, Y.; Xie, W. -F.; Su, Z. Manipulating Phosphorescence Efficiencies of Orange Iridium(III) Complexes through Ancillary Ligand Control. Dyes Pigm. 2019, 160, 119-127.

(7) Hwong, F. M.; Chen, H. -Y.; Chen, P. -S.; Liu, C. -S.; Chi, Y.; Shu, C. -F.; Wu, F.; Chou, P. -T.; Peng, S. -M.; Lee, G. H. Iridium Complexes with Orthometalated Quinoxaline Ligands: Subtle Tuning of Emission to the Saturated Red Color. Inorg. Chem. 2005, 44, 1344-1353. 
(8) Song, Y.-H.; Yeh, S. -J.; Chen, C. -T.; Chi, Y.; Liu, C. -S.; Yu, J. -K.; Hu, Y. -H.; Chou, P. -T.; Peng, S. -M.; Lee, G. -H. Bright and Efficient, Non-doped, Phosphorescent Organic Red-Light-Emtting Diodes. Adv. Funct. Mater. 2004, 14, 1221-1226.

(9) Gao, J.; You, H.; Fang, J.; Ma, D.; Wang, L.; Jing, X.; Wang, F. Pure Red Electrophosphorescent Organic Light-Emitting Diodes Based on a New Iridium Complex. Synth. Met. 2005, 155, 168-171.

(10) Kim, H. U.; Jang, H. J.; Choi, W.; Park, S.; Park, T.; Lee, J. Y.; Bejoymohandas, K. S. Aggregation-Induced Phosphorescence Enhancement in Deep-Red and Near-Infrared Emissive Iridium(III) Complexes for Solution-Processable OLEDs. J. Mater. Chem. C 2020, 8, 4789-4800.

(11) Kesarkar, S.; Mróz, W.; Penconi, M.; Pasini, M.; Destri, S.; Cazzaniga, M.; Ceresoli, D.; Mussini, P. R.; Baldoli, C.; Giovannella, U.; Bossi, A. Near-IR Emitting Iridium(III) Complexes with Heteroaromatic B-Diketonate Ancillary Ligands for Efficient SolutionProcessed OLEDs: Structure-Property Correlations. Angew. Chem. Int. Ed. 2016, 55, 27142718. 